\title{
A Generalized Kinetic Model for Compartmentalization of Organometallic Catalysis
}

\author{
Brandon J. Jolly ${ }^{1}$, Chong Liu ${ }^{1,2 *}$ \\ ${ }^{1}$ Department of Chemistry and Biochemistry, University of California, Los Angeles, California \\ 90095, United States \\ ${ }^{2}$ California NanoSystems Institute (CNSI), University of California, Los Angeles, Los Angeles, \\ CA 90095, USA
}

* To whom correspondence may be addressed. Email: chongliu@chem.ucla.edu 


\begin{abstract}
Compartmentalization is an attractive approach to enhance catalytic activity by retaining reactive intermediates and mitigating deactivating pathways. Such a concept has been well explored in biochemical and more recently, organometallic catalysis to ensure high reaction turnovers with minimal side reactions. However, a scarcity of theoretical framework towards confined organometallic chemistry impedes a broader utility for the implementation of compartmentalization. Herein, we report a general kinetic model and offer design guidance for a compartmentalized organometallic catalytic cycle. In comparison to a non-compartmentalized catalysis, compartmentalization is quantitatively shown to prevent the unwanted intermediate deactivation, boost the corresponding reaction efficiency $(\gamma)$, and subsequently increase catalytic turnover frequency (TOF). The key parameter in the model is the volumetric diffusive conductance $\left(F_{V}\right)$ that describes catalysts' diffusion propensity across a compartment's boundary. Optimal values of $F_{V}$ for a specific organometallic chemistry are needed to achieve maximal values of $\gamma$ and TOF. Our model suggests that a tailored compartment design, including the use of nanomaterials, is needed to suit a specific organometallic catalytic cycle. This work provides justification and design principles for further exploration into compartmentalizing organometallics to enhance catalytic performance.
\end{abstract}




\section{INTRODUCTION}

Compartmentalization has been well documented in biochemical literature as one method for achieving efficient in vivo tandem catalysis by encapsulating enzymes in well-defined microand nano-structures. ${ }^{1-7}$ By controlling the diffusion of species in and out of compartment boundaries, nature is able to retain reactive or toxic intermediates, increase local substrate concentration, and mitigate deactivating or competing pathways. ${ }^{1-7}$ For example, carboxysome microcompartments enhance the rate of $\mathrm{CO}_{2}$ fixation by encapsulating the cascade of carbonic anhydrase and ribose 1,5-bisphosphate carboxylase/oxygenase to generate high local concentration of $\mathrm{CO}_{2}$ and exclude deactivating $\mathrm{O}_{2}$ within their polyhedral structures. ${ }^{8,9}$ Also, the last two steps of tryptophan biosynthesis - the conversion of indole-3-glycerol-phosphate to indole and then to tryptophan - takes advantages of the substrate-channeling effect bestowed by compartmentalized subunits of tryptophan synthase. ${ }^{10,11}$ Here, a hydrophobic tunnel between the two subunits retains the indole intermediate, which prevents its free diffusion and participation in deactivating side reactions. ${ }^{10}$ With billions of years of evolution, compartmentalization appears the mainstay of biology to manage the complex network of biochemical reactions that are frequently competing and incompatible with each other in a homogenous solution.

The success of natural compartmentalized enzyme cascades inspires the development of bio-mimetic synthetic catalysis with organometallic chemistry being the latest frontier. Multiple groups have employed well-defined spatial organization at the nano- and microscopic levels to construct in vitro biocatalytic and organometallic cascades with enhanced catalytic performance. ${ }^{2,3,12-16}$ Encapsulating NiFe hydrogenase in virus capsids improves its proteolytic and thermal stability as well as enhances the rate of $\mathrm{H}_{2}$ production. ${ }^{12}$ Confining a biochemical cascade of $\beta$-galactose, glucose oxidase, and horse radish peroxidase in metal-organic frameworks led to an enhancement of reaction yield in comparison to a freely diffusing analogue. ${ }^{13,14}$ The extent to which reaction yields are enhanced in confined enzyme cascades is reported to correlate with the distance between active sites, suggesting that spatial organization or localization of catalysts is beneficial in tandem or cascade reactions. ${ }^{15}$ In addition to biocatalysis, recently compartmentalization of organometallic catalysts has been experimentally demonstrated. ${ }^{17-23}$ For example, our group employed a nanowire-array electrode to pair seemingly incompatible $\mathrm{CH}_{4}$ activation based on $\mathrm{O}_{2}$-sensitive rhodium (II) metalloradical ( $\mathrm{Rh}(\mathrm{II})$ ) with $\mathrm{O}_{2}$-based oxidation for 
$\mathrm{CH}_{3} \mathrm{OH}$ formation. ${ }^{17,24}$ The application of a reducing potential to the nanowire array electrode created a steep $\mathrm{O}_{2}$ gradient within the wire array electrode, such that an anoxic compartment was established at the bottom of the wires. As a result that was not observable for planar electrode without an anoxic region, a catalytic cycle was formed in which the air-sensitive $\mathrm{Rh}(\mathrm{II})$ activated $\mathrm{CH}_{4}$ in the $\mathrm{O}_{2}$-free region of the wire array electrode, while $\mathrm{CH}_{3} \mathrm{OH}$ synthesis proceeded in the aerobic domain with $\mathrm{O}_{2}$ as the terminal electron acceptor. The retainment of the ephemeral $\mathrm{Rh}(\mathrm{II})$ intermediate by the nanowire electrode for catalytic $\mathrm{CH}_{4}$-to- $\mathrm{CH}_{3} \mathrm{OH}$ conversion ${ }^{17,24}$ encourages us to further explore the design principles of compartmentalizing organometallic cascades for higher turnovers with mitigated deactivation pathways.

We envision that a theoretical framework for organometallic catalysis will expand the use of compartmentalization for organometallic chemistry. In biochemistry, mathematical modeling of confined enzyme cascades has been well developed and offers the design principles in natural systems ${ }^{11,25}$ and for engineered bio-compartments. ${ }^{11,16,25,26}$ The models pinpoint a key parameter, volumetric diffusive conductance $\left(F_{V}\right)$, which describes the diffusion propensity across a compartment's boundary. $F_{V}$ is determined by a compartment's surface-to-volume ratio and its boundary's permeability. ${ }^{26,27}$ An optimal value of $F_{V}$ tailored to the specific biochemical reactions are needed in order to achieve better reactivity in comparison to the non-compartmentalized alternative. Similarly, we contend that further development of compartmentalized organometallic chemistry demands a similar quantitative design principle. In a model organometallic cycle that includes oxidative addition (OA), isomerization/migratory insertion (Iso/MI), and reductive elimination (RE) along with undesirable deactivation pathways, ${ }^{28}$ what are the suitable values of the compartment's physical parameters for minimal deactivation and maximal turnover frequency (TOF) (Figure 1)? Unfortunately, there has been a paucity of theoretical treatment for this question despite the exciting progresses in experimental demonstration. ${ }^{17-23}$ Such a lack of theoretical treatment motivates us to establish a general kinetic model and quantitatively investigate how compartmentalization will affect the competing reaction pathways and the corresponding turnover of the desired organometallic catalysis.

Here we report a general kinetic model and offer design guidance for a compartmentalized organometallic catalytic cycle. We took advantage of the established theoretical frameworks in biochemistry ${ }^{16,25,26}$ and applied such kinetic frameworks to a model compartmentalized 

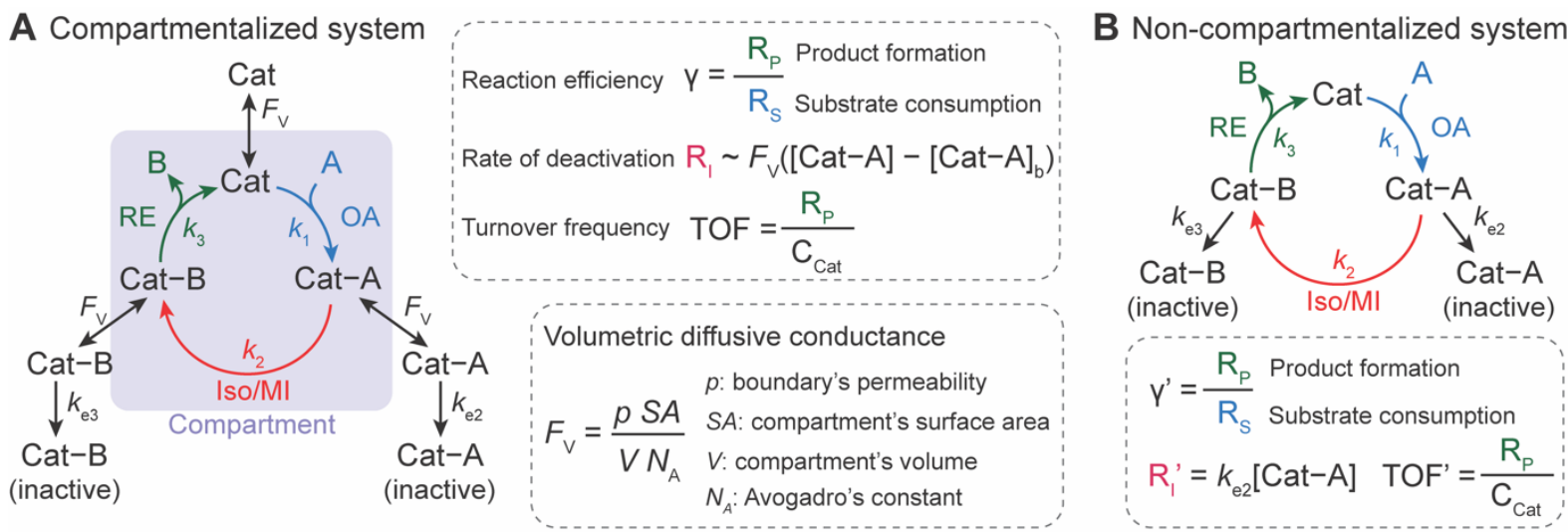

Figure 1. Schematic of a general compartmentalized catalytic cycle in organometallic chemistry (A) and the corresponding non-compartmentalized (freely diffusing) system (B). $\gamma$, reaction efficiency; $R_{S}$, rate of substrate consumption; $R_{I}$, rate of intermediate elimination; $R_{P}$, rate of product formation; TOF , turnover frequency; $F_{V}$, volumetric diffusive conductance; $p$, compartment boundary's permeability for catalytic intermediates; $S A$, compartment's surface area; $V$, compartment volume; $N_{A}$, Avogadro's constant; OA, oxidative addition (rate constant $k_{1}$ ); Iso/MI, isomerization/migratory insertion (rate constant $k_{2}$ ) in conjunction with a competing deactivation (rate constant $k_{e 2}$ ); RE, reductive elimination (rate constant $k_{3}$ ) in conjunction with a competing deactivation (rate constant $k_{e 3}$ ).

organometallic cycle with competing deactivation pathways (Figure 1A), ${ }^{28}$ and a noncompartmentalized counterpart as a control scenario (Figure 1B). Under assumptions and simplifications applicable to organometallic catalysis, as a proof-of-concept we examined three important metrics of this catalytic cycle in both compartmentalized and non-compartmentalized scenarios: (1) reaction efficiency $(\gamma)$ that gauges the percentage of intermediates funneled towards desirable catalytic turnover over deactivation pathways; (2) the deactivating rate of intermediate Cat - A $\left(R_{I}\right)$; (3) the turnover frequency (TOF) that measures the steady-state catalytic rate despite intermediate deactivation. When compartments' $F_{V}$ values are smaller than the intrinsic kinetics of the organometallic cycle in question, a compartmentalized system can significantly outperform a homogeneous counterpart with respect to $\gamma$ and TOF with a lower value of $R_{I}$. We illustrated the general relationship for specific organometallic catalysis to achieve maximal $\gamma$ and TOF. The established kinetic model can be adapted to suit a plethora of catalytic cycles with synthetic compartments, offering a framework to be expanded on for advanced compartmentalization of chemical catalysis. 


\section{RESULTS AND DISCUSSION}

Establishing a general kinetic framework of compartmentalization for an organometallic catalytic cycle

Our investigation starts with a hypothetical three-step organometallic cycle confined within a compartment in conjunction with multiple deactivation pathways in the exterior bulk solution (Figure 1A and Supplementary Information Section 1A). ${ }^{28}$ Catalytic species Cat of a presumed constant concentration in the bulk ([Cat $]_{b} \equiv C_{c a t}$ ) diffuses into the compartment of volume $V$ and bind substrate molecule $A$ through oxidative addition to form intermediate species $C a t-A$, either pseudo-first-order $(\mathrm{m}=1)^{29-32}$ or pseudo-second-order $(\mathrm{m}=2)^{24,33,34}$ with respect to Cat (rate constant $k_{1}$ ). After a step of isomerization or migratory insertion (rate constant $k_{2}$ ) converts Cat $A$ species to the product adduct $C a t-B$, the catalytic cycle is completed by the reductive elimination that transforms $C a t-B$ back to $C a t$ with the release of product $B$ (rate constant $k_{3}$ ). Here we presume that $C a t, C a t-A$ and $C a t-B$ intermediates all can diffuse across the compartment boundary and there are two possible competing deactivation pathways in the homogenous solution outside the compartment. The deactivations of Cat $-A$ and $C a t-B$ are presumed pseudo-first-order with respect to the intermediates with rate constants $k_{e 2}$ and $k_{e 3}$, respectively. Similarly, a non-compartmentalized system was constructed for the sake of comparison with the same set of kinetic reaction parameters (Figure 1B and Supplementary Information Section 1B). The established compartmentalized and non-compartmentalized catalytic cycles are generally applicable to a broad range of organometallic catalysis with concurrent deactivation processes ${ }^{28,35-37}$, which has been well reviewed and comprehensively discussed in literature. ${ }^{36}$

For the compartmentalized scenario (Figure 1A), we additionally assign volumetric diffusive conductance $\left(F_{V}\right)$ to quantitatively describe the extent of mass transport, predominantly diffusion-based, between the compartment and the surrounding bulk solution (Supplementary Information Section 2). As a measure of molecules' propensities to diffusively cross the compartment's boundary under a given concentration gradient, $F_{V}$ is defined as the product of compartment boundary's permeability $(p)$ and its total surface area $(S A)$ while divided by Avogadro's constant $\left(N_{A}\right)$ and the volume $(V)$ of the corresponding compartment (Figure 1A). ${ }^{26}$ In particular, $p$ is proportional to the species' diffusion coefficients $(D)$ and inversely proportional 
to the distance of diffusion path across the boundary. ${ }^{38}$ In our analysis, we assume $p$ remains constant for Cat, Cat $-A$ and $C a t-B$, given the fact that the catalytic center are frequently more bulky in comparison to the substrate/product, and the catalytic intermediates typically have similar diffusion coefficients despite the reaction-related adducts. We also assume that substrate $A$ and product $B$ are small enough that faster diffusion of $A$ and $B$ leads to minimal concentration gradients for $A$ and $B$. Under such assumptions, a single value of $F_{V}$ for the catalytic intermediates is sufficient to describe the effect of compartmentalization on a catalytic cycle. Because the value of $p$ depends on the compartment's physical properties, the design of compartment's surface-tovolume ratio $(S A / V)$ and materials' properties at the compartment's boundary has significant impacts on the value of $F_{V}$, and subsequently the overall catalytic turnover as will be discussed in this study.

In this work we aim to study the steady-state phenomena of compartmentalized catalysis. We assume constant, time-independent concentrations of Cat, Cat $-A$ and Cat $-B$ in both the compartment ([Cat], $[\mathrm{Cat}-\mathrm{A}]$, and $[\mathrm{Cat}-B]$, respectively) as well as the surrounding bulk solution $\left([C a t]_{b} \equiv C_{c a t}\right.$ (vide supra), $[C a t-A]_{b}$ and $[C a t-B]_{b}$, respectively). Similarly, in the bulk solution substrate $A$ is maintained at a constant concentration $\left(C_{A}\right)$ and fast removal of product $B$ is ensured $([B] \rightarrow 0)$. Such assumptions including $[C a t]_{b} \equiv C_{c a t}$ pertain to a flow reactor with sufficient amount of catalysts or a batch reaction under high catalyst loading and low conversion (Supplementary Information Section 1). Alternatively, a constant total catalyst concentration including all catalytic species in the bulk can be presumed $\left(C_{\text {Cat,total }} \equiv[\mathrm{Cat}]_{b}+[\mathrm{Cat}-\mathrm{A}]_{b}+\right.$ $[\text { Cat }-B]_{b}=$ constant), Supplementary Information Section 3). We have analyzed the catalysis under both sets of assumptions. We note that the latter set of assumptions with a constant total catalyst concentration, more complicated to solve mathematically and labeled as "model

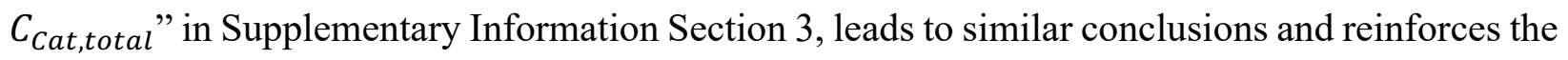
general applicability of the following results solved when we assume $[\mathrm{Cat}]_{b} \equiv C_{c a t}$. Unless noted specifically, the results discussed below will be based on the former set of assumptions (Supplementary Information Section 1).

A set of steady-state kinetic equations are constructed to reflect both the compartmentalized and non-compartmentalized scenarios (equations S1-S5 and S67-S69) for an organometallic catalytic cycle following the analysis protocols established in biochemistry. ${ }^{26}$ 
Comparing to the non-compartmentalized case that only includes reactions in the homogenous solution (equation S67-69), the equations for the compartmentalized case (equation S1-5) additionally consider the reactions in the compartment as well as the mass transport across the boundary, whose magnitudes are governed by both the value of $F_{V}$ and the concentration gradients across the compartment's boundary. Detailed mathematical treatment of the established equations can be found in Supplementary Information Section 1 and a few key outputs of the model are evaluated here. As one of the proposed benefits of compartmentalization is the capability of retaining reactive intermediates within the compartment without significant catalyst deactivation in the bulk, $2,3,14,16$ we are interested in evaluating the steady-state consumption rate of substrate $A$ $\left(R_{S}\right)$, the generation rate of product $\mathrm{B}\left(R_{P}\right)$, and the deactivation rate of intermediates $C a t-A\left(R_{I}\right)$ (Figure 1A). Moreover, in both compartmentalized and non-compartmentalized scenarios, we aim to analyze the rate of reaction, numerically represented as turnover frequency TOF, and the efficacy of transforming the substrate $A$ into targeted product $B$, numerically represented as reaction efficiency $\gamma$ that is defined as the percentage of intermediates funneled towards desirable catalytic turnover. ${ }^{16,26}$ In both cases, $\gamma$ is calculated as the ratio between the formation rate of product $B$ and the consumption rate of substrate $A$. In the case of pseudo-first-order kinetics towards Cat in oxidative addition $(\mathrm{m}=1), \gamma, R_{I, m=1}$, and $T O F_{m=1}$ in a compartmentalized system can be expressed as,

$$
\begin{aligned}
& \gamma=\frac{k_{2} k_{3}}{\left(f_{2}+k_{2}\right)\left(f_{3}+k_{3}\right)} \\
& R_{I, m=1}=\frac{k_{1} k_{e 2} F_{V}^{2} C_{C a t} C_{A}}{\left(a_{1}+F_{V}\right)\left(f_{2}+k_{2}\right)\left(F_{V}+k_{e 2}\right)} \\
& T O F_{m=1}=\frac{k_{1} k_{2} k_{3} F_{V} C_{A}}{\left(a_{1}+F_{V}\right)\left(f_{2}+k_{2}\right)\left(f_{3}+k_{3}\right)}
\end{aligned}
$$

in which,

$$
\begin{aligned}
& f_{2}=\frac{F_{V} k_{e 2}}{F_{V}+k_{e 2}} \text { and } f_{3}=\frac{F_{V} k_{e 3}}{F_{V}+k_{e 3}} \\
& a_{1}=k_{1} C_{A}-\frac{k_{1} k_{2} k_{3} C_{A}}{\left(f_{2}+k_{2}\right)\left(f_{3}+k_{3}\right)}
\end{aligned}
$$

In comparison under a non-compartmentalized scenario, the corresponding $\gamma^{\prime}, R_{I, m=1}^{\prime}$, and $T O F_{m=1}^{\prime}$ are expressed as, 
$\gamma^{\prime}=\frac{k_{2} k_{3}}{\left(k_{2}+k_{e 2}\right)\left(k_{3}+k_{e 3}\right)}$

$R_{I, m=1}^{\prime}=\frac{k_{1} k_{e 2} C_{C a t} C_{A}}{k_{2}+k_{e 2}}$

$T O F_{m=1}^{\prime}=\frac{k_{1} k_{2} k_{3} C_{A}}{\left(k_{2}+k_{e 2}\right)\left(k_{3}+k_{e 3}\right)}$

The mathematical expressions for $\gamma, R_{I}$, and TOF results, derived from Supplementary Information Sections 1 and 3 for the assumptions outlined in the main text and for model $C_{\text {Cat,total }}$ respectively, are summarized in Table S1-S2 as a reference. The successful construction and mathematical derivation of a general kinetic model in organometallic catalysis warrants quantitative evaluation about the efficacy of compartmentalization under different reaction kinetics and compartment properties.

\section{Exemplary numerical comparisons between compartmentalized and non- compartmentalized catalysis}

The derived analytical solutions to the established kinetic model allow us to numerically calculate the values of $\gamma, R_{I}$, and TOF in both compartmentalized and non-compartmentalized scenarios. Specifically, we set out to evaluate under what conditions compartmentalization is beneficial with a given set of parameters pertaining to the compartment's properties and kinetics of organometallic reactions. As an introductory example representative to a typical organometallic catalytic cycle, we assume that $C_{\mathrm{cat}}=1 \mathrm{mM}$ and $C_{\mathrm{A}}=10 \mathrm{mM}$, as organometallic catalytic systems often operate near $10 \mathrm{~mol} \%$ catalyst loading. ${ }^{35}$ Values of kinetic parameters are $k_{1} \in\left[10^{-5}, 10^{4}\right]$ $\mathrm{M}^{-1} \cdot \mathrm{s}^{-1}(\mathrm{~m}=1)^{29-32}$ or $k_{1} \in\left[10^{-3}, 10^{6}\right] \mathrm{M}^{-2} \cdot \mathrm{s}^{-1}(\mathrm{~m}=2) ; ;^{17,24,33,34} k_{2} \in\left[10^{-3}, 10^{6}\right] \mathrm{s}^{-1} 39,40$ and $k_{3}=$ $10^{6} \mathrm{~s}^{-1} .{ }^{41,42}$ When either $k_{1}$ or $k_{2}$ is not a variable of interest, they are set as $k_{1}=0.1 \mathrm{M}^{-1} \cdot \mathrm{s}^{-1}(\mathrm{~m}=$ 1) or $10 \mathrm{M}^{-2} \cdot \mathrm{s}^{-1}(\mathrm{~m}=2)$ and $k_{2}=10^{3} \mathrm{~s}^{-1} \cdot 29-32$ The selection of those kinetic parameters is based on reviews of oxidative addition and migratory insertion, as well as reported kinetic studies using techniques such as time resolved infrared spectroscopy for transient species on the intermediates during carbonylation and $\mathrm{O}_{2}$ reduction and transfer, among others. ${ }^{17,24,29-34,39,40}$ The selection of $k_{3}$ parameter value implicitly assumes fast reductive elimination from $C a t-B$, which is supported by the observation that reductive eliminations are often not the rate-determining step in a catalytic cycle. $^{41,42}$ The values of deactivation kinetics $k_{e 2}$ for $C a t-A$ and $k_{e 3}$ for $C a t-B$ are selected 
with additional assumptions, given the dearth of reported kinetic values for the less exciting deactivation steps. As the reductive elimination from $C a t-B$ is sufficiently fast, our primary focus is to examine the deactivation from $\mathrm{Cat}-\mathrm{A}$ hence how the comparison between $k_{2}$ and $k_{e 2}$ will affect the overall catalysis. Subsequently we assign $k_{e 3}=k_{3}=1 \times 10^{6} \mathrm{~s}^{-1}$ so that the rate of competing deactivation from $C a t-B$ is no lower than rate of reductive elimination. Similarly, when $k_{e 2}$ is not a variable of interest, we set $k_{e 2}=k_{2}=1 \times 10^{3} \mathrm{~s}^{-1}$ to match the kinetics of isomerization/migratory insertion. Last, we set $F_{V} \in[30,600] \mathrm{s}^{-1}$, whose range is estimated based on the diffusion coefficient of $9 \times 10^{-10} \mathrm{~m}^{2} \cdot \mathrm{s}^{-1}$ from tabulated organometallic catalysts, ${ }^{43,44}$ as well as the geometry and properties of reported microscopic compartments in porous materials, supramolecular assemblies, nanoscopic micelles, and the use of nanowire array electrode in our previous work (see Supplementary Information Section 2). ${ }^{17-20,45-47}$ Overall, our selection of kinetic values here represents an organometallic catalytic cycle whose oxidative addition step is turnover-limiting and the deactivation of yielded Cat $-A$ intermediate is the most critical issue, while the fast reductive elimination leaves the deactivation of $C a t-B$ species secondary in terms of $\gamma$ and TOF. With varying values of $F_{V}$ and changing ratios between the values of $k_{2}$ and $k_{e 2}$, the trend of compartmentalization's efficacy can be unveiled.

The numerically calculated values of $\gamma, R_{I}$, and TOF as a function of $k_{2}$ and $F_{V}$ illustrate that compartmentalization generally outperforms the non-compartmentalized scenarios with a higher tolerance towards undesirable deactivation reactions (Figure 2). Under a fixed rate constant of deactivation $\left(k_{e 2}=1 \times 10^{3} \mathrm{~s}^{-1}\right)$ and pseudo-first-order oxidative addition $\left(k_{1}=0.1 \mathrm{M}^{-1} \cdot \mathrm{s}^{-1}\right.$ for $\mathrm{m}=1$ ), values of $\gamma, R_{I}$, and TOF in a compartmentalized system are plotted as a function of both $k_{2}$ and $F_{V}$ in Figure $2 \mathrm{~A}$ to $2 \mathrm{C}$. The rate of oxidative addition $\left(k_{2}\right)$ is understandably a predominant factor in all three plots. When $k_{2}$ is much smaller than the rate of deactivation $\left(k_{e 2}\right), \gamma$ approaches zero (Figure 2A) when the deactivation of Cat $-A$ outcompetes the step of isomerization/migratory insertion, which is concurrent with a higher rate of deactivation $\left(R_{\mathrm{I}}\right.$ in Figure 2B) and lower TOF value (Figure 2C). Alternatively, when $k_{2}$ is much larger than $k_{e 2}$ and the deactivation step is less relevant, $\gamma$ plateaus towards unity with concomitant increase in TOF (Figure 2A,C). Despite the dominant role of $k_{2}$, whether or not the system is compartmentalized strongly affects the values of $\gamma, R_{I}$, and TOF (Figure 2D to $2 \mathrm{~F}$ ). While the trend is generally applicable for all values of $F_{V}$, a specific case $\left(F_{V}=320 \mathrm{~s}^{-1}\right)$ that corresponds to the nanowire array 

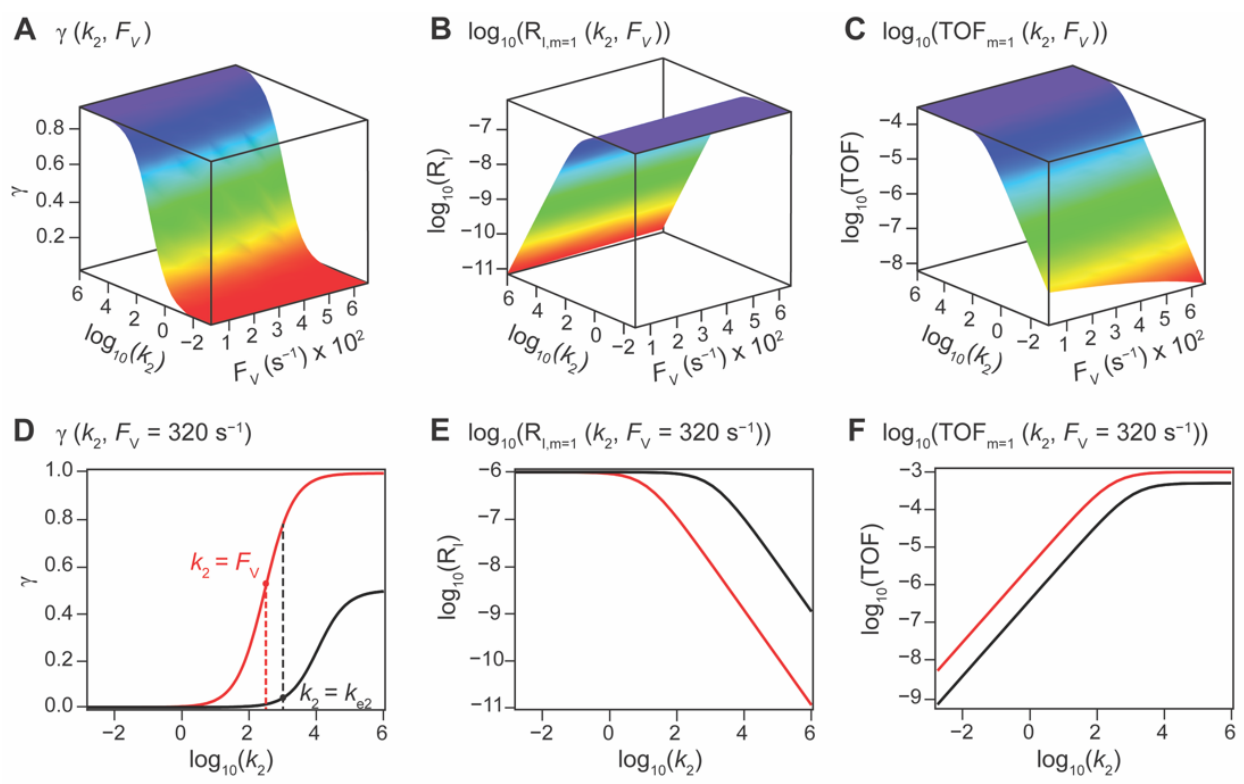

— Compartmentalized Non-compartmentalized

Figure 2. Reaction efficiency $(\gamma, \mathbf{A}$ and $\mathbf{D})$, logarithmic of intermediate outflux rate $\left(\log _{10}\left(R_{I}\right)\right.$, $\mathbf{B}$ and $\mathbf{E}$ ), and logarithmic of turnover frequency $\left(\log _{10}(T O F), \mathbf{C}\right.$ and $\left.\mathbf{F}\right)$ as a function of volumetric diffusive conductance $\left(F_{V}\right)$ and logarithmic of the rate constant for isomerization or migratory insertion (Iso/MI) $\left(\log _{10}\left(k_{2}\right)\right)$. A to $\mathbf{C}$, compartmentalized scenario depicted in Figure 1A. D to $\mathbf{F}$, comparisons between compartmentalized (red trace, when $F_{V}=320 \mathrm{~s}^{-1}$ ) and noncompartmentalized (black trace) scenarios. $\mathrm{m}=1, k_{1}=0.1 \mathrm{M}^{-1} \cdot \mathrm{s}^{-1}$ notwithstanding $\mathbf{A}$ and $\mathbf{D}, k_{e 2}$ $\left.=1 \times 10^{3} \mathrm{~s}^{-1}\right), k_{3}=k_{e 3}=1 \times 10^{6} \mathrm{~s}^{-1}$. The selection of those exemplary values is based on literature reports on the kinetics of relevant organometallic systems (vide supra).

electrode for $\mathrm{CH}_{4}$-to- $\mathrm{CH}_{3} \mathrm{OH}$ conversion in our previous work, ${ }^{17}$ illustrates under which situation the advantages of compartmentalization will be observed. As the value of $k_{2}$ increases, the compartmentalized scenario observes an increase of reaction efficiency $\gamma$ in a sigmoidal fashion when $k_{2}$ approaches the value of $F_{V}$ (red trace in Figure 2D); in contrast, $\gamma$ in a noncompartmentalized case (black trace in Figure 2D) won't increase until $k_{2}$ approaches the value of $k_{e 2}$. Similarly, with $F_{V} \ll k_{e 2}$ and under a reasonably large value of $k_{2}$, compartmentalization suppresses the rate of deactivation $R_{I}$ (Figure 2E) and increases the TOF by roughly no less than one order of magnitude (Figure 2F). Evaluations assuming pseudo-second-order kinetics towards Cat in the step of oxidative addition $(\mathrm{m}=2)$ lead to the same conclusion (Figures S3A and S4A). Those observations suggest that the strategy of compartmentalization allows a catalytic cycle to be much more tolerant towards undesirable side reactions, as long as $F_{V}$ is much smaller than $k_{e 2}$ $\left(F_{V} \ll k_{e 2}\right)$ with a judicious compartment design. 
Additional examination suggests that a less "leaky" compartment with smaller $F_{V}$ value should be more effective. The difference of $\gamma$ values between compartmentalization and noncompartmentalization is the biggest when $F_{V}<k_{2} \ll k_{e 2}$ (Figure 2D). Such a trend is more apparent when $\gamma, R_{I}$, and TOF were plotted as a function of $F_{V}$ under fixed values of $k_{2}$ and $k_{e 2}$ (Figure $3 \mathrm{~A}$ to $3 \mathrm{C}$ ). In both situations when $\mathrm{m}=1$ and $\mathrm{m}=2$, a larger value of $F_{V}$ leads to smaller values of $\gamma$ and TOF and large value of $R_{I}$. This suggests that a more "leaky" compartment is not sufficient to conserve the yielded intermediates and is more prone to deactivation. A similar conclusion can be obtained when investigating the dependence of $\gamma, R_{I}$, and TOF as a function of $F_{V}$ and $k_{e 2}$ (Figure 3D to 3F, Figure S3B and S4B). Significant decrease of $\gamma$ and increase of $R_{I}$ was observed at high $F_{V}$ values, particularly when the values of $k_{e 2}$ are so large that the deactivation is much faster and intermediate $C a t-A$ has a much shorter life time once it diffuses out of the compartment.

The above noted observations can be mathematically rationalized from our derived equations. When the value of $F_{V}$ is similar to or even larger than $k_{e 2}$ or $k_{e 3}\left(F_{V} \gtrsim k_{e 2}\right.$ or $\left.k_{e 3}\right)$,

$\left.f_{2}\right|_{F_{V} \gtrsim k_{e 2}}=\frac{F_{V} k_{e 2}}{F_{V}+k_{e 2}}=k_{e 2} \quad$ and $\left.\quad f_{3}\right|_{F_{V} \gtrsim k_{e 3}}=\frac{F_{V} k_{e 3}}{F_{V}+k_{e 3}}=k_{e 3}$

This will lead to $\gamma \approx \gamma^{\prime}$, i.e. the reaction efficiency is not significantly altered with compartmentalization in comparison to the non-compartmentalized case.

Alternatively, when $F_{V} \ll k_{e 2}$ or $k_{e 3}$, we have

$\left.f_{2}\right|_{F_{V} \ll k_{e 2}}=\frac{F_{V} k_{e 2}}{F_{V}+k_{e 2}}=F_{V} \quad$ and $\left.\quad f_{3}\right|_{F_{V} \ll k_{e 3}}=\frac{F_{V} k_{e 3}}{F_{V}+k_{e 3}}=F_{V}$

This leads to

$\left.\gamma\right|_{F_{V} \ll k_{e 2} \& k_{e 3}}=\frac{k_{2} k_{3}}{\left(f_{2}+k_{2}\right)\left(f_{3}+k_{3}\right)}=\frac{k_{2} k_{3}}{\left(F_{V}+k_{2}\right)\left(F_{V}+k_{3}\right)}=\frac{1}{\left(\frac{F_{V}}{k_{2}}+1\right)\left(\frac{F_{V}}{k_{3}}+1\right)}$
$\left.\operatorname{TOF}_{m=1}\right|_{F_{V} \ll k_{e 2} \& k_{e 3}}=\frac{k_{1} k_{2} k_{3} F_{V} C_{A}}{\left(k_{1} C_{A}-\frac{k_{1} k_{2} k_{3} C_{A}}{\left(F_{V}+k_{2}\right)\left(F_{V}+k_{3}\right)}+F_{V}\right)\left(F_{V}+k_{2}\right)\left(F_{V}+k_{3}\right)}$ 

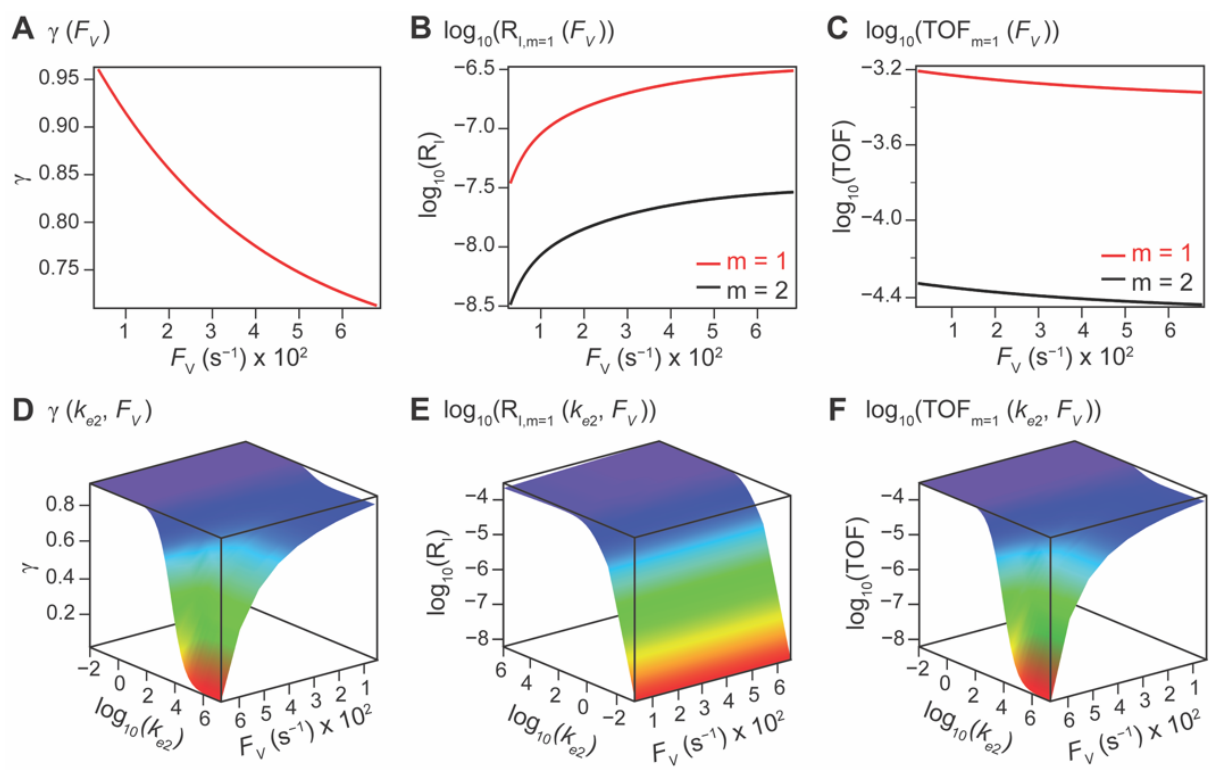

$\mathbf{F} \log _{10}\left(\mathrm{TOF}_{\mathrm{m}=1}\left(k_{e 2}, F_{v}\right)\right)$

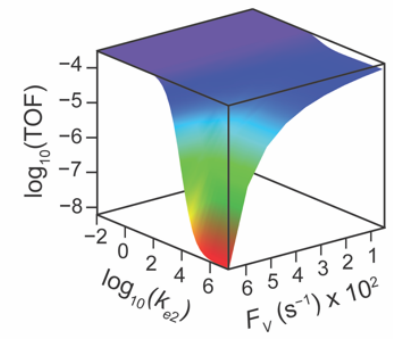

Figure 3. In a compartmentalized system, reaction efficiency ( $\gamma, \mathbf{A}$ and $\mathbf{D})$, logarithmic of intermediate outflux rate $\left(\log _{10}\left(R_{I}\right), \mathbf{B}\right.$ and $\left.\mathbf{E}\right)$, and logarithmic of turnover frequency $\left(\log _{10}(T O F), \mathbf{C}\right.$ and $\left.\mathbf{F}\right)$ as a function of volumetric diffusive conductance $\left(F_{V}\right)$ and logarithmic of the rate constant for $C a t-A$ deactivation $\left(\log _{10}\left(k_{e 2}\right)\right)$. The axis of $F_{V}$ and $\log _{10}\left(k_{e 2}\right)$ in $\mathbf{D}$ and $\mathbf{F}$ are inverted for the sake of presentation clarity. $k_{1}=0.1 \mathrm{M}^{-1} \cdot \mathrm{s}^{-1}$ and $10 \mathrm{M}^{-2} \cdot \mathrm{s}^{-1}$ for $\mathrm{m}=1$ and 2 , respectively, notwithstanding A and D. $k_{2}=k_{e 2}=1 \times 10^{3} \mathrm{~s}^{-1} \cdot k_{3}=k_{e 3}=1 \times 10^{6} \mathrm{~s}^{-1}$.

The equations noted above suggest that optimal, near-unity reaction efficiency $\gamma$, high $T O F$, and low $R_{I}$ values would be obtained when $F_{V} \ll k_{2}$ and $k_{3}$, which is consistent with our observations in Figure 2.

Lastly, when a constant total catalyst concentration including all catalytic species in the bulk is presumed ("model $C_{\text {Cat,total }}$ ", $C_{\text {Cat,total }} \equiv[\text { Cat }]_{b}+[\text { Cat }-A]_{b}+[\text { Cat }-B]_{b}=$ constant, Supplementary Information Section 3), the calculated values of $R_{I}$ and TOF as functions of $F_{V}$ and $k_{2}$ displayed little difference to the above-mentioned observations (Figures S5 to S7), while the derived expressions of $\gamma$ are identical under both assumptions (Table S1 and S2). Such observations suggest that slight variation in the assumptions of the developed model does not significantly alter how the compartment's $F_{V}$ impacts the kinetics of overall catalysis.

\section{Implication on the design of compartmentalized catalysis}

The established kinetic model and the numerical evaluation offers an affirmative answer to the efficacy of compartmentalized organometallic catalysis and, if needed, what is the desired 
properties of the established compartment. When the rate constants of the steps in the catalytic cycle $\left(k_{2}\right.$ and $\left.k_{3}\right)$ are commensurate with or greater than the rate constants of deactivation steps ( $k_{e 2}$ and $k_{e 3}$ ), i.e. $k_{2} \gtrsim k_{e 2}$ and $k_{3} \gtrsim k_{e 3}$, compartmentalization is not necessary since the intrinsic reactivity of catalysis is sufficiently fast with respect to undesirable side reactions. Compartmentalization should be considered under $k_{2}<k_{e 2}$ and $k_{3}<k_{e 3}$, when the intrinsic reactivities of the catalytic cycle cannot outcompete the deactivation pathways. The efficacy of compartmentalization will be observable, as long as the compartment's volumetric diffusive conductance $F_{V}$ is much smaller than $k_{e 2}$ or $k_{e 3}\left(F_{V} \ll k_{e 2}\right.$ or $\left.k_{e 3}\right)$. Nonetheless, one interesting conclusion from our analysis is that maximal efficacy of compartmentalization (reaction efficiency $\gamma \rightarrow 1)$ demands $F_{V}$ to be smaller not only than the rate constants of deactivation steps $\left(k_{e 2}\right.$ and $\left.k_{e 3}\right)$ but also than the rate constants of steps in the catalytic cycle $\left(k_{2}\right.$ and $\left.k_{3}\right)$. This requirement for maximal $\gamma$ stems from the fact that a "leaky" compartment with large $F_{V}$ is not sufficient to conserve the yielded intermediates and is prone to deactivation. Practically, such a requirement is indeed a blessing for organometallic chemistry. As typical organometallic studies do not commonly characterize the deactivating side reactions, there lacks detailed kinetic information the values of $k_{e 2}$ or $k_{e 3}$ in comparison to the knowledge about catalytic kinetics $\left(k_{2}\right.$ and $\left.k_{3}\right)$. Because we posit that criteria of $F_{V}<k_{2}$ and $F_{V}<k_{3}$ are sufficient for a compartment to "revive" a catalytic cycle unfunctional in a homogenous solution, kinetic information of the in-cycle steps $\left(k_{2}\right.$ and $\left.k_{3}\right)$ is sufficient for future design of functional compartmentalization.

The feasibility of obtaining the range of $F_{V}$ from the kinetics of the proposed catalytic cycle offers more guidance for the materials design for the compartment. As $F_{V}$ is proportional to the compartment boundary's permeability $(p)$ and its surface-to-volume ratio $(S A / V),{ }^{26}$ multiple synthetic handles could be applied to achieve a desirable $F_{V}$ value. A less permeable interface at the boundary of compartment as well as smaller surface-to-volume ratio will help to reduce the mass transport hence the value of $F_{V}$. Characterization techniques that help determine encapsulation geometry and assess permeability, such as electron microscopies and chromatographic methods, should be welcomed for more detailed mechanistic investigations in experimental demonstration. ${ }^{48-51}$ One interesting result from this argument is that a compartment of extremely small dimension, for example of nanoscopic scale, may not be necessarily beneficial, because nanoscopic dimensions with its large surface-to-volume ratio tends to create a "leaky" 
compartment. Careful compartment design tailored to the specific kinetics of the catalytic cycle is recommended before experimental implementation.

Last, we cautioned that our established model only considers the mass transport of catalysts and assumes an unconditionally fast supply of substrate $A$ and quick removal of product $B$. While such assumptions have their real-life correspondence under certain circumstances (vide supra), the currently established model is incapable of accounting for the possible mass-transport limitation for the substrate and product, which could be induced by a small $F_{V}$ value recommended by the model presented here. Given that, we cautioned that a lower bound of $F_{V}$ exists for optimal performance in practical applications, and an unnecessarily small value of $F_{V}$ could be detrimental to the compartment design.

\section{CONCLUSION}

In this study, we developed a general kinetic framework for compartmentalizing organometallic catalysis with competing deactivation reactions in the bulk solution. Compartmentalization is only necessary under $k_{2}<k_{e 2}$ and $k_{3}<k_{e 3}$, when the intrinsic reactivities of the catalytic cycle cannot outcompete the deactivation pathways. Under such situations, the kinetic model predicts that careful compartment design with suitable values of volumetric diffusive conductance $\left(F_{V}<k_{2}\right.$ and $\left.F_{V}<k_{3}\right)$ is capable of achieving maximal reaction efficiency $(\gamma)$ and turnover frequency (TOF). It is intriguing that the kinetics of deactivation steps are not needed for the design of compartment, as long as it is known that $k_{2}<k_{e 2}$ and $k_{3}<k_{e 3}$. A tailored compartment design, including the use of nanomaterials, is needed to suit a specific organometallic catalysis. Such insights will assist in future a priori design of compartmentalized organometallics for enhanced catalytic performance. 


\section{ASSOCIATE CONTENT}

\section{Supporting Information}

The Supporting Information is available at DOI:

Mathematical derivation of compartmentalized and non-compartmentalized kinetic models, tables of solutions to key reaction metrics, and additional figures.

\section{Author Information}

C. L. supervised the project. B. J. J. performed the mathematical derivations for the theoretical framework. All the authors discussed the results and assisted during the manuscript preparation.

Corresponding Author

*Email: chongliu@chem.ucla.edu

ORCID

Brandon J. Jolly: 0000-0003-4991-5212

Chong Liu: 0000-0001-5546-3852

\section{Notes}

The authors declare no competing financial interest.

\section{ACKNOWLEDGEMENTS}

C. L. acknowledges the NSF Award (CHE-2027330), startup fund from the University of California, Los Angeles, and the financial support of the Jeffery and Helo Zink Endowed Professional Development Term Chair. 


\section{TABLE OF CONTENTS}

How to design compartments for an organometallic cycle?

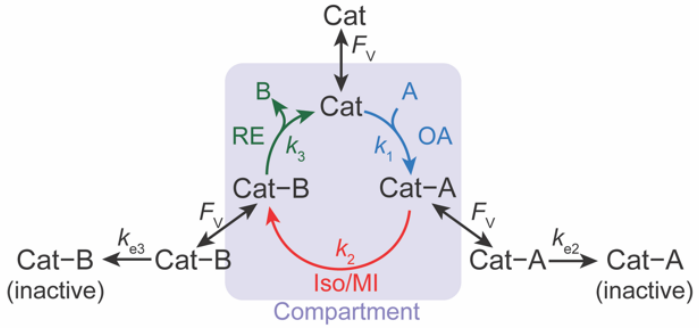




\section{REFERENCES}

1. S. Hurtley, Science, 2009, 326, 1205-1205.

2. A. H. Chen and P. A. Silver, Trends Cell Biol., 2012, 22, 662-670.

3. U. Jandt, C. You, Y. H. P. Zhang and A. P. Zeng, Adv. Biochem. Eng. Biotechnol., 2013, 137, 41-65.

4. A. Zecchin, P. C. Stapor, J. Goveia and P. Carmeliet, Curr. Opin. Biotechnol., 2015, 34, 73-81.

5. J. L. Avalos, G. R. Fink and G. Stephanopoulos, Nat. Biotechnol., 2013, 31, 335-341.

6. B. P. Tu, Science, 2005, 310, 1152-1158.

7. M. T. Alam, V. Olin-Sandoval, A. Stincone, M. A. Keller, A. Zelezniak, B. F. Luisi and M. Ralser, Nat. Commun., 2017, 8, 1-13.

8. G. C. Cannon, C. E. Bradburne, H. C. Aldrich, S. H. Baker, S. Heinhorst and J. M. Shively, Appl. Environ. Microbiol., 2001, 67, 5351-5361.

9. B. D. Rae, B. M. Long, L. F. Whitehead, B. Förster, M. R. Badger and G. D. Price, J. Mol. Microbiol. Biotechnol., 2013, 23, 300-307.

10. K. S. Anderson, E. W. Miles and K. A. Johnson, J. Biol. Chem., 1991, 266, 8020-8033.

11. K. S. Chavan and S. Calabrese Barton, J. Phys. Chem. C, 2018, 122, 14474-14480.

12. P. C. Jordan, D. P. Patterson, K. N. Saboda, E. J. Edwards, H. M. Miettinen, G. Basu, M. C. Thielges and T. Douglas, Nat. Chem., 2016, 8, 179-185.

13. W.-H. Chen, M. Vázquez-González, A. Zoabi, R. Abu-Reziq and I. Willner, Nat. Catal., 2018, 1, 689-695.

14. M. Vázquez-González, C. Wang and I. Willner, Nat. Catal., 2020, 3, 256-273.

15. O. Idan and H. Hess, ACS Nano, 2013, 7, 8658-8665.

16. Y. Zhang and H. Hess, ACS Catal., 2017, 7, 6018-6027.

17. B. S. Natinsky, S. Lu, E. D. Copeland, J. C. Quintana and C. Liu, ACS Cent. Sci., 2019, 5, 1584-1590.

18. S. H. A. M. Leenders, R. Gramage-Doria, B. De Bruin and J. N. H. Reek, Chem. Soc. Rev, 2015, 44, 433-448.

19. J. Lu, J. Dimroth and M. Weck, J. Am. Chem. Soc., 2015, 137, 12984-12989.

20. T. M. Rayder, A. T. Bensalah, B. Li, J. A. Byers and C.-K. Tsung, J. Am. Chem. Soc., 2021, 143, 1630-1640.

21. R. W. Simms and M. F. Cunningham, Macromolecules, 2008, 41, 5148-5155.

22. N. M. B. Smeets, J. P. A. Heuts, J. Meuldijk, M. F. Cunningham and A. M. Van Herk, Macromolecules, 2009, 42, 7332-7341.

23. K. Wang, J. H. Jordan, X. Y. Hu and L. Wang, Angew. Chem. Int. Ed., 2020, 59, 1371213721.

24. B. B. Wayland, S. Ba and A. E. Sherry, J. Am. Chem. Soc., 1991, 113, 5305-5311.

25. F. Hinzpeter, U. Gerland and F. Tostevin, Biophys. J., 2017, 112, 767-779.

26. S. Tsitkov and H. Hess, ACS Catal., 2019, 9, 2432-2439.

27. H. C. Berg, Random Walks in Biology, Princeton University Press, 1993.

28. G. Miessler and D. Tarr, Inorganic Chemistry, Upper Saddle River, NJ: Pearson Education Inc., 2004.

29. J. A. Labinger, Organometallics, 2015, 34, 4784-4795.

30. K. L. Breno, M. D. Pluth, C. W. Landorf and D. R. Tyler, Organometallics, 2004, 23, $1738-$ 1746. 
31. P. R. Ellis, J. M. Pearson, A. Haynes, H. Adams, N. A. Bailey and P. M. Maitlis, Organometallics, 1994, 13, 3215-3226.

32. J. H. Espenson, O. Pestovsky, P. Huston and S. Staudt, J. Am. Chem. Soc., 1994, 116, 28692877.

33. T. D. Ju, R. F. Lang, G. C. Roper and C. D. Hoff, J. Am. Chem. Soc., 1996, 118, 53285329.

34. Z. M. Heiden and T. B. Rauchfuss, J. Am. Chem. Soc., 2007, 129, 14303-14310.

35. D. Astruc, Organometallic chemistry and catalysis, Springer, 2007.

36. R. H. Crabtree, Chem. Rev., 2015, 115, 127-150.

37. A. Meißner, E. Alberico, H.-J. Drexler, W. Baumann and D. Heller, Catal. Sci. Technol., 2014, 4, 3409-3425.

38. W. Stein, Transport and diffusion across cell membranes, Elsevier Inc., 1986.

39. P. C. Ford and S. Massick, Coord. Chem. Rev., 2002, 226, 39-49.

40. S. M. Massick and P. C. Ford, Organometallics, 1999, 18, 4362-4366.

41. P. Nilsson and O. F. Wendt, J. Organomet. Chem., 2005, 690, 4197-4202.

42. L. Jin, H. Zhang, P. Li, J. R. Sowa Jr and A. Lei, J. Am. Chem. Soc., 2009, 131, 9892-9893.

43. P. S. Pregosin, P. G. A. Kumar and I. Fernández, Chem. Rev., 2005, 105, 2977-2998.

44. D. Drago, P. S. Pregosin and A. Pfaltz, Chem. Commun., 2002, 286-287.

45. B. S. Natinsky, B. J. Jolly, D. M. Dumas and C. Liu, Chem. Sci., 2021, 12, 1818-1825.

46. T. A. Bender, M. Morimoto, R. G. Bergman, K. N. Raymond and F. D. Toste, J. Am. Chem. Soc., 2019, 141, 1701-1706.

47. P. Qu, M. Kuepfert, M. Hashmi and M. Weck, J. Am. Chem. Soc., 2021, 143, 4705-4713.

48. C. Huang, C. Liu, X. Chen, Z. Xue, K. Liu, X. Qiao, X. Li, Z. Lu, L. Zhang, Z. Lin and T. Wang, Adv. Sci., 2020, 7, 1903180.

49. W. Li, Y. Zhang, Z. Xu, Q. Meng, Z. Fan, S. Ye and G. Zhang, Angew. Chem. Int. Ed., 2016, 55, 955-959.

50. L. Lei, Q. Zhang, S. Shi and S. Zhu, Langmuir, 2017, 33, 6108-6115.

51. E. Haldoupis, T. Watanabe, S. Nair and D. S. Sholl, ChemPhysChem, 2012, 13, 3449-3452. 